\title{
Balkanologie
}

Balkanologie Revue d'études pluridisciplinaires

Vol. VIII, $n^{\circ} 1 \mid 2004$

Volume VIII Numéro 1

\section{La Macédoine du Pirin en Bulgarie communiste}

Politiques d'Etat et ethnicité

Pirin Macedonia in Communist Bulgaria: State policy and ethnicity

Tchavdar Marinov

\section{CpenEdition}

Journals

Édition électronique

URL : https://journals.openedition.org/balkanologie/530

DOI : 10.4000/balkanologie.530

ISSN : 1965-0582

Éditeur

Association française d'études sur les Balkans (Afebalk)

Édition imprimée

Date de publication : 1 juin 2004

ISSN : 1279-7952

Référence électronique

Tchavdar Marinov, «La Macédoine du Pirin en Bulgarie communiste », Balkanologie [En ligne], Vol. VIII, $\mathrm{n}^{\circ} 1$ | 2004, mis en ligne le 21 janvier 2010, consulté le 28 juin 2022. URL : http://

journals.openedition.org/balkanologie/530 ; DOI : https://doi.org/10.4000/balkanologie.530

(C) Tous droits réservés 


\title{
LA MACEDOINE DU PIRIN EN BULGARIE COMMUNISTE : POLITIQUES D'ETAT ET ETHNICITÉ
}

\author{
Tchavdar Marinov*
}

D'innombrables ouvrages (encyclopédies, dictionnaires géographiques, brochures) sur les minorités nationales en Bulgarie contemporaine font état de quelques 200 à 250000 " Macédoniens ethniques " dans ce pays, en général " dépourvus " de possibilités d'expression de leur appartenance nationale et de leur " culture spécifique "1. Des cas d'" infractions aux droits de l'Homme " commises par les autorités à l'égard de citoyens bulgares se considérant d'origine ethnique macédonienne retentirent à travers des ONG comme Bulgarian Helsinki Committee et Amnesty International. Récemment, la Bulgarie fut même condamnée par la Cour européenne de Strasbourg pour avoir violé l'Article 11 de la Convention européenne des droits de l'Homme sur cinq points ${ }^{2}$. En janvier 2004, Sofia fut de nouveau critiquée par la Commission contre le racisme et l'intolérance auprès du Conseil de l'Europe pour " attitude discriminatoire " envers les représentants de la minorité macédonienne traditionnellement qualifiée par des politiciens bulgares de " minorité fantôme ". Après la chute du régime communiste en Bulgarie, apparurent divers partis politiques composés de militants pour la reconnaissance de l'identité ethnique macédonienne. Ces derniers, dont le plus important, OMO " Ilinden ", restent illégaux. Au bout d'un an d'existence légale, la branche modéré OMO

• Doctorant à l'EHESS, Paris. (tchavdarmarinov@yahoo.fr).

${ }^{1}$ Cf. Ivanov (Mihail), "On the Macedonian Authenticity ", New Balkans Politics , (6), (accessible en ligne $\mathrm{http}: / /$ www.newbalkanpolitics.org.mk/OldSite/Issue 6/ivanov.macedonian.eng.asp), qui se réfère à la recherche de Michel Foucher, publiée par le Conseil de l'Europe en 1994, et à l'article sur la Bulgarie de l'encyclopédie éditée par le Minorities' Rights Group International.

${ }^{2}$ Le cas Stankov and the United Macedonian Organization Ilinden v. Bulgaria, décision du 2 octobre 2001, disponible sur http://hudoc.echr.coe.int/hudoc. Sur les problèmes des Macédoniens en Bulgarie - voir les rapports annuels du Bulgarian Helsinki Committee sur http://www.bghelsinki.org ainsi que l'étude : http://www.greekhelsinki.gr/pdf/cedime-se-bulgaria-macedonians.PDF. 
" Ilinden "-Pirin fut déclarée, en février 2000, anti-constitutionnelle, son activité étant jugée " séparatiste "3. Les nationalistes macédoniens dénoncent la politique de la Bulgarie contemporaine dont l'élite académique reproduit la position idéologique, selon laquelle les personnes revendiquant une identité nationale macédonienne, y compris celles de l'Ancienne République Yougoslave de Macédoine, sont " en vérité des Bulgares ethniques " parlant une forme standardisée d'un dialecte ouest-bulgare.

À présent, une partie importante de la population bulgare est composée de descendants de réfugiés de la Macédoine ottomane (fin XIX ${ }^{\mathrm{e}}$ - début $\mathrm{XX}^{\mathrm{e}}$ siècle) ainsi que de descendants d'expatriés venus après les Guerres balkaniques et la Première Guerre mondiale de la "Macédoine du Vardar " annexée par la Serbie ou de la "Macédoine d'Égée " rattachée à la Grèce4. Dans le paysage politique actuel, ces descendants soutiennent souvent l'irrédentisme bulgare contemporain vis-à-vis de la république ex-yougoslave de Macédoine voire de la région du même nom en Grèce actuelle ; ils sont souvent aussi actifs au sein des partis nationalistes bulgares comme la VMRO-SMD/BND5. Des historiens indiquent, en outre, la difficulté de faire une distinction " objective " ou " culturelle " entre " Bulgares " et " Macédoniens " en Bulgarie : ces derniers parlent, en général, la même langue - le bulgare standard, ils ont à peu près les mêmes références historiques, ce qui, aux yeux de certains spécialistes, transforme les Macédoniens de Bulgarie en une « identité minoritaire essentielle-

3 Voir son site http://www.omoilindenpirin.org/. Cf. Poulton (Hugh), Who are the Macedonians ?, Bloomington / Indianapolis : Indiana University Press, 2000, pp. 151-159. Les spécialistes bulgares publièrent très peu d'études sur l'influence du " macédonisme ", ou bien de l'ethnonationalisme macédonien, en Bulgarie - un sujet toujours estimé " délicat " : Rusanov (Valerij), Tomova (Ilona), " Makedonizmăt v Bălgarija - dve izsledvanija ", in Aspekti na etnokulturnata situacija v Bălgarija, Sofia : Asociacija AKSES, 1994 ; Stoyanova-Boneva (Bonka), Nikolov (Stephan E.), "In Search of "Bigfoot" : Competing Identities in Pirin Macedonia, Bulgaria ", in Roudometof (Victor), ed., The Macedonian Question : Culture, Historiography, Politics, New York : Columbia University Press, 2000 ; Stoyanova-Boneva (Bonka), " Ethnicity in Pirin Macedonia : Blurred Categories, Emergent Minority ", Balkanistica, 9, 1994-1996; Bakalova (Maria), " Bulgarian "Macedonian" Nationalism in the Post 1989 Decade ", New Balkan Politics, (6), Skopje - http://www.newbalkanpolitics.org.mk/OldSite/Issue_6/bakalova.natio.eng.asp .

4 Des hauts fonctionnaires du gouvernement bulgare comme Mihail Ivanov estiment à 2 millions les citoyens bulgares " originaires n de Macédoine dont " la majorité préservèrent une forte conscience bulgare " - un chiffre qui reste cependant contestable de même que le contenu exact du concept d' " originaire de Macédoine ". Il faut souligner que, depuis le XIXe siècle et jusqu'à la période de l'Entre-deux-guerres, l'identité macédonienne n'était pas forcément exclusive vis-à-vis de l'auto-identification ethnique bulgare et, souvent, toutes les deux " cohabitèrent " sous des formes différentes. Une longe série de mythomoteurs nationaux (personnages et événements historiques, symboles etc.) sont revendiqués à la fois par les Bulgares et les Macédoniens contemporains.

5 Sur la place de la VMRO dans la vie politique bulgare contemporaine - voir Stoyanova-Boneva (Bonka), Nikolov (Stephan E.), art.cit.; Nikolov (Stephan E.), « The Bulgarian Obsession : Macedonia in the XX " $^{\text {th }}$ Century Bulgarian Politics ", New Balkan Politics, (1), disponible à l'adresse suivante : http://www.newbalkanpolitics.org.mk/OldSite/issue_1/tekst.asp?id=nikolov_eng . 
ment subjective ${ }^{6}$. Pourtant, des revendications ethniques macédoniennes se font entendre en Bulgarie au moins depuis soixante ans.

Cet article est consacré à certains aspects de la politique du Parti communiste et de l'État bulgare entre 1944 et 1989 vis-à-vis de l'identité ethnique macédonienne dans la région du Pirin 7 - la seule partie appartenant à la Bulgarie que l'on appelle la " Macédoine géographique ". Cette analyse est effectuée sur la base de documents des archives centrales et départementales du Parti (du Politbureau et du Secrétariat du Comité central, des " sections " au sein du Comité central, du Bureau du Comité départemental à Blagoevgrad, etc.) et des archives de différentes institutions de l'État (Administration Centrale de Statistique et d'autres). La plupart des documents sont cités et exploités ici pour la première fois. Faisant partie de l'information " classée ", les données sur ce sujet, gardées aux archives du ministère de l'Intérieur à Sofia, sont malheureusement toujours inaccessibles. Les résultats de la recherche documentaire sont également comparés avec les données d'entretiens réalisés au mois d'avril 2004 avec des militants nationalistes macédoniens en Bulgarie.

\section{STATISTIQUES ET POLITIQUES D’ÉTAT}

Le peuple du Pirin même (...) acceptait les instituteurs comme s'ils étaient ses fils et frères, et ses enfants apprenaient, avec une avidité rare, leur langue et leur histoire. Travailler avec une telle jeunesse - c'était à la fois noble et gratifiant. La gratitude émanait des cœurs des jeunes... ${ }^{8}$

Telles étaient les intentions yougoslaves, mais au cours de l'opération visant à une incorporation (priobštavane) totale de la Macédoine du Pirin (...) les libraires et les instituteurs de Skopje firent face à la résistance des élèves, des enseignants, de la population en général. Le refus d'accepter les valeurs " culturelles " allogènes et très suspicieuses était absolu. En fin de compte, l'échec total de l'entreprise macédoniste est tout à fait naturel...9

${ }^{6}$ Expression appartenant à Lory (Bernard), L'Europe balkanique de 1945 à nos jours, Paris : Ellipses, 1996, p. 138.

7 " Pirin n est le nom de la montagne traversant la région et la divisant en deux parties.

${ }^{8}$ Mitrev (Dimitar), Pirinska Makedonija, Skopje : Naša kniga, 1986, p. 267.

9 Angelov (Veselin), Hronika na edno nacionalno predatelstvo, Blagoevgrad : Universitetsko izdatelstvo, 1999, p. 299. 
Ces deux extraits, dont le premier tiré de l'ouvrage d'un historien littéraire macédonien et le second d'une étude historiographique bulgare, devraient en principe décrire le même événement dans le même espace géographique. Il s'agit de l'activité d'enseignants de langue macédonienne ainsi que de la mise en place d'une série d'institutions culturelles macédoniennes qui ont lieu en 1946-1948 dans la région du Pirin. Néanmoins, les deux textes cités donnent des interprétations différentes des mêmes événements. Si l'analyste macédonien décrit ce processus comme un rétablissement de la " justice historique " accepté avec enthousiasme par la population " purement macédonienne " de la région, son collègue bulgare voit ce même moment historique comme un " crime " impardonnable du Parti communiste bulgare ${ }^{10}$ qui, de concert avec Belgrade et Moscou, amorça une " assimilation forcée " de la population " patriotique bulgare " du Pirin. Correspondant au département administratif de Blagoevgrad ${ }^{11}$, la Macédoine du Pirin fit l'objet d'une série de négociations et de controverses avec la Yougoslavie titiste et d'une politique intérieure active pendant le régime communiste en Bulgarie. Cette politique changea à plusieurs reprises et parfois de manière radicale. Ces deux extraits historiographiques relèvent d'une vaste littérature consacrée à la première phase de la "Question macédonienne " en Bulgarie communiste : la période 1944-1948, lorsque, conformément aux projets d'une fédération bulgaro-yougoslave, le dirigeant du Parti communiste et du gouvernement bulgare Georgi Dimitrov conféra un statut spécial à la région du Pirin. Celui-ci représenta une sorte d'" autonomie culturelle " (enseignement en macédonien, librairies et théâtre macédonien) censée préparer la région à une réunification prochaine à la république yougoslave de Macédoine conformément aux résolutions du $10^{\mathrm{e}}$ Plénum du Comité central du Parti en 1946 et aux accords passés avec la Yougoslavie à Bled en $1947^{12}$.

En 1946, fut effectué le premier recensement en Bulgarie qui atteste la présence de "Macédoniens ". Celui-ci donne le nombre de 169544 Macédoniens dont 160641 dans la région du Pirin déclarant une " nationalité " macédo-

10 Jusqu'en décembre 1948, nommé Parti ouvrier bulgare (communistes) ou BRP (k), puis Parti communiste bulgare (BKP).

${ }^{11}$ En 1949, la division administrative " région " (oblast) fut remplacée par " département " (okrăg). La ville principale Gorna Džumaja fut en 1950 rebaptisée à Blagoevgrad d'après le nom du fondateur du premier parti marxiste en Bulgarie Dimităr Blagoev. En 1949-1950, deux villes du département reçurent des noms de révolutionnaires macédoniens - Nevrokop devint Goce Delčev et Sveti Vrač-Sandanski. En 1987, le département de Blagoevgrad fut inclus dans la région de la capitale Sofia. Depuis 1999, Blagoevgrad est de nouveau centre de " région ".

12 Sur cette période, les études les plus représentatives pour les visions bulgares et macédoniennes contemporaines sont, respectivement, l'ouvrage de Angelov (Veselin), op.cit. (lui-même, originaire du Pirin) et la monographie de Jotevski (Vasil), Nacionalnata afirmacija na Makedoncite vo Pirinskiot del na Makedonija 1944-1948, Skopje : INI, 1966. 
nienne, le concept de nationalité (narodnost) correspondant plutôt à la notion française d' "origine ethnique "13. Il faut remarquer que, dans les statistiques non-publiées, 132010 de ces Macédoniens du Pirin ont été enregistrés sous la rubrique "langue de communication " (govorim ezik) le bulgare contre 28611 la " langue macédonienne ". Les données sur les nationalités de 1946 ne furent rendues publiques qu'en 1970, mais les Macédoniens antérieurement recensés ont été effacés des statistiques et ajoutés au nombre total des Bulgares ${ }^{14}$. Entre-temps, la rupture Tito-Staline de 1948 avait mis fin aux projets de fédération bulgare-yougoslave et, par la suite, à l'" autonomie " ou à la " macédonisation " culturelle du Pirin. Les instituteurs et les libraires macédoniens de Skopje furent obligés de quitter la Bulgarie. À partir de son $16^{\mathrm{e}}$ Plénum et $5^{\mathrm{e}}$ Congrès en 1948, la direction du Parti révisa sa politique de "concessions " dans la région du Pirin sans toutefois remettre en question l'appartenance nationale macédonienne de sa population. Mais les statistiques sur le nombre de Macédoniens du premier recensement étaient au fur et à mesure dénoncées par la direction du Parti qui reconnut avoir réalisé une série de trucages lors du recensement.

Plusieurs documents d'archives du Parti publiés par la suite par les historiens bulgares sont venus confirmer que le nombre des Macédoniens de 1946 avait été dans une large mesure "fabriqué " et qu'il avait peu à voir avec l'auto-identification réelle de la population. Le secrétaire de Parti du district (okolija) de Petrič fit l'aveu suivant : " Au cours du recensement, on a fait pression (natisk). Le Comité régional nous a obligés à enregistrer 60 à $70 \%$ de Macédoniens dans notre district. Nous, on a pressé un peu plus (pozasilihme rabotata) et on a eu $90 \%$ de Macédoniens "15. À la veille du recensement, les dirigeants locaux envoyèrent des dépêches aux maires des cinq districts (okolii) de la région précisant que toute la population devait être enregistrée comme " macédonienne à l'exception des Juifs, des Tsiganes, des Turcs et des Bulgares venus de [l'intérieur de] la Bulgarie. Les Bulgares musulmans [bălgaro-mohamedanite] doivent être recensés aussi comme Macédoniens... " 16 . En revanche, l'absence de directives claires quant à l'enregistrement de la

\footnotetext{
${ }^{13} \mathrm{Au}$ recensement de 1956 , on utilisa le terme nacionalnost, alors que les autres recensements de l'époque communiste - ceux de 1946,1965 et 1975, emploient le terme traditionnel narodnost. On peut consulter les données sur les "Macédoniens " aux archives centrales de l'État (Centralen däržaven arhiv, ci-après- CDA, F. 210, OP. 1, A.E. 764, A.E. 860). Comme la case "Macédoniens " ne figure pas sur les formulaires initiaux, les données sont ajoutées à la main. La plupart des autres documents sur le recensement de 1946 ne distinguent pas les "Macédoniens " des « Bulgares ".
}

14 Voir Rezultati ot prebrojavaneto na naselenieto na 31.XII.1946, DUI pri MS, Sofia, 1970, p. 16.

15 Archives Centrales du Parti (Centralen partien arhiv), ci-après CPA, F. 1, OP. 5, A.E. 21, L. 13. Cité sans références exactes chez Nikolov (Stephan E.), "The Bulgarian Obsession n (art.cit.).

16 Cf. Angelov (Veselin), op.cit., p. 134. Pour consulter d'autres documents de ce genre-Ibid., p. 125-144. 
"langue de communication " pourrait expliquer que la grande majorité de "Macédoniens " parle le " bulgare ".

Les historiens bulgares sont cependant loin de démontrer que la " macédonisation " du Pirin provoquait une indignation ou une résistance massive ${ }^{17}$ : les sources documentaires attestent plutôt d'une inertie relative de la part de la population et même de cas d'un certain intérêt ou d'une curiosité envers les innovations de l'" autonomie culturelle ". Par exemple, la librairie macédonienne à Gorna Džumaja était souvent visitée par les gens locaux qui n'achetaient cependant pas les livres ${ }^{18}$. Mais loin de susciter l'enthousiasme revendiqué par les historiens de Skopje, la politique menée dans le Pirin jusqu'en 1948 n'excluait pas non plus certains cas de mécontentement et d'opposition. Les difficultés d'apprentissage de la langue standard macédonienne - souvent estimée trop " serbisée "(sărbiziran)-sont parmi les causes les plus fréquentes de mécontentement ${ }^{19}$. En 1948, les secrétaires des comités locaux du Parti confessèrent : " Quant à la langue [macédonienne], on voyait que les gens n'avaient pas de volonté de l'étudier car elle ne leur était pas familière, pourtant on insistait et, sur la base de directives (merki) administratives, on les contraignait à l'apprendre... " ${ }^{20}$. Même si le Parti organisa des réunions pour clarifier le " besoin " d'apprendre la langue macédonienne, "les personnes présentes ne semblaient pas convaincues de ce qu'on leur disait $n^{21}$.

Pourtant, dix ans plus tard, c'est-à-dire après la fin de la politique d'" autonomie culturelle " du Pirin, le deuxième recensement de la population donnait un chiffre non négligeable de Macédoniens voire plus élevé qu'en 1946. En 1956, leur nombre total se monte à 187789 personnes en Bulgarie dont 178862 en Macédoine du Pirin ${ }^{22}$.

${ }^{17}$ Nẻanmoins prétendue par l'historien bulgare Mičev qui, pour la démontrer, se réfère surtout aux réactions des parties politiques d'opposition (Mičev (Dobrin), Makedonskijat văpros i bălgaro-jugoslavskite otnošenija (9 septemvri 1944-1949), Sofia : Universitetsko izdatelstvo, 1994, pp. 251-263).

${ }^{18}$ CPA, F. 1, OP. 15, A.E. 160, L. 40.

19 Voir CPA, F. 1, OP. 6, A.E. 364 , L. 14 de 1947 ; CPA, F. 1, OP. 5, A.E. 21 d'avril 1948 - L. 1-9, L. 15 ; CPA, F. 1, OP. 13 , A.E. 55, L. 2, L. 20 de juillet-août 1948 ; CPA, F. 1, OP. 13, A.E. 112, L. 3-4, L. 8, L. 28 d'août 1948. Même les gens qui achetaient des livres en macédonien les comprenaient difficilement-CPA, F. 1, OP. 15, A.E. 249, L. 19 de février-mars 1948.

${ }^{20}$ CPA, F. 1, OP. 13 , A.E. 55 , L. 27.

${ }^{21}$ CPA, F. 1, OP. 13, A.E. 112, L. 8.

${ }^{22}$ Prebrojavane na naselenieto $v$ NRB na 1.XII.1956. Obšti rezultati, kn. II, Sofia : CSU, 1960, pp. 106, p. 110, p. 214-230. 


\section{Recensement de 1946}

\begin{tabular}{|l|c|c|c|}
\hline & Macédoine & Bulgarie & Total \\
\hline Région (oblast) & $\mathbf{1 6 0 6 4 1}$ & $\mathbf{5 4 4 2 5}$ & $\mathbf{2 5 2} \mathbf{5 7 5}$ \\
GORNA DŽUMAJA & $(63,6 \%)$ & $(21,5 \%)$ & $(100 \%)$ \\
\hline District (okolija) & $\mathbf{2 4 1 6 9}$ & $\mathbf{2 4} \mathbf{8 2 5}$ & $\mathbf{5 1 3 8 3}$ \\
GORNA DŽUMAJA & $(47 \%)$ & $(48,3 \%)$ & $(100 \%)$ \\
\hline District & $\mathbf{2 9 3 5 1}$ & $\mathbf{1 4} 007$ & $\mathbf{6 5 0 8 2}$ \\
NEVROKOP & $(45,1 \%)$ & $(21,5 \%)$ & $(100 \%)$ \\
\hline District & $\mathbf{4 2} \mathbf{0 4 7}$ & $\mathbf{2 9 2 7}$ & $\mathbf{4 6 1 6 1}$ \\
PETRIČ & $(91,1 \%)$ & $(6,3 \%)$ & $(100 \%)$ \\
\hline District & $\mathbf{2 3} \mathbf{8 2 7}$ & $\mathbf{5 0 6 6}$ & $39 \mathbf{7 2 5}$ \\
RAZLOG & $(60 \%)$ & $(12,8 \%)$ & $(100 \%)$ \\
\hline District & $\mathbf{4 1 2 4 7}$ & $\mathbf{7 6 0 0}$ & $\mathbf{5 0 2 2 4}$ \\
SVETI VRAČ & $(82,1 \%)$ & $(15,1 \%)$ & $(100 \%)$ \\
\hline
\end{tabular}

Recensement de 1956

\begin{tabular}{|l|c|c|c|}
\hline & Macédoine & Bulgarie & Total \\
\hline Département (okrăg) & $\mathbf{1 7 8} \mathbf{8 6 2}$ & $\mathbf{9 3 6 7 1}$ & $\mathbf{2 8 1} \mathbf{0 1 5}$ \\
BLAGOEVGRAD & $(63,6 \%)$ & $(33,3 \%)$ & $(100 \%)$ \\
\hline District & $\mathbf{3 0 5 6 1}$ & $\mathbf{2 6 4 0 3}$ & $\mathbf{5 8 5 6 1}$ \\
BLAGOEVGRAD & $(52,2 \%)$ & $(45,1 \%)$ & $(100 \%)$ \\
\hline District & $\mathbf{2 9 5 6 8}$ & $\mathbf{3 1} \mathbf{9 4 1}$ & $\mathbf{6 4 5 9 0}$ \\
GOCE DELČEV & $(45,8 \%)$ & $(49,5 \%)$ & $(100 \%)$ \\
\hline District & $\mathbf{4 0 0 0 8}$ & $\mathbf{7 3 3 4}$ & $\mathbf{4 8 3 8 4}$ \\
PETRIČ & $(82,7 \%)$ & $(15,2 \%)$ & $(100 \%)$ \\
\hline District & $\mathbf{2 3 3 5 2}$ & $\mathbf{2 3 3 4 4}$ & $\mathbf{4 7} 603$ \\
RAZLOG & $(49,1 \%)$ & $(49 \%)$ & $(100 \%)$ \\
\hline District & $\mathbf{5 5 3 7 3}$ & $\mathbf{4 6 4 9}$ & $\mathbf{6 1 8 7 7}$ \\
SANDANSKI & $(89,5 \%)$ & $(7,5 \%)$ & $(100 \%)$ \\
\hline
\end{tabular}

Tableau 1 : Habitants de " nationalité macédonienne "et de " nationalité bulgare " dans la région du Pirin (Région de Gorna Džumaja, puis Département de Blagoevgrad) selon les recensements de 1946 et de 1956. Le total comprend aussi les pourcentages des autres nationalités recensées. Sources : CDA, F. 210, OP. 1, A.E. 764, L. 1-5; CDA, F. 210, OP. 1, A.E. 860, L. 1-7 ; Prebrojavane na naselenieto v NRB na 1.XII.1956 g., Obšti rezultati, kn. II, CSU pri MS, Sofia, 1960, p. 106.

À l'exception des districts de Blagoevgrad (anciennement Gorna Džumaja) et de Sandanski (Sveti Vrač), on voit une tendance nette à l'augmentation du nombre et du pourcentage de " Bulgares ", les Macédoniens demeurant toutefois majoritaires, sauf dans le district de Goce Delčev (Nevrokop). Une baisse considérable des Bulgares est à remarquer dans le district de Sandanski. Les arguments de spécialistes bulgares sur ces données ne sont guère convaincants. 
Les historiens de Sofia et de Blagoevgrad ${ }^{23}$ n'apportent pas de preuves d'une manipulation administrative directe en 1956. Bien au contraire, les directives officielles du Parti pour la conduite du deuxième recensement insistent sur l'erreur de 1946 et demandent à ce que ceux qui furent antérieurement recensés comme Macédoniens se déclarent " tels qu'ils se sentent (čuvstvat) " ${ }^{24}$. Le Parti a ainsi révisé, dans une certaine mesure, la politique de " macédonisation " mais sans initier de mesures pratiques en faveur de la " re-bulgarisation "de la région du Pirin. Cela provoqua des perplexités même chez les fonctionnaires locaux du Parti comme attesté par la déclaration d'un de ces fonctionnaires en novembre 1956 :

... à l'époque, lorsqu'on effectuait le recensement précédent, il y avait un tas de gens qui venaient chez moi pour me demander de les enregistrer comme Bulgares et [pour me dire] qu'on ne devait pas les enregistrer comme Macédoniens. Mais comme il y avait une telle pression et c'était la politique du Parti, on devait les recenser comme Macédoniens. Le problème (bedata) est d'avoir été une fois enregistrés comme Macédoniens, alors que maintenant on doit se déclarer Bulgares...25

Or, sur la reconnaissance théorique de l'identité macédonienne, la politique officielle, elle, ne fut guère différente de celle d'avant 1948. Le Parti communiste bulgare continua à reconnaître l'existence d'une nationalité macédonienne, distincte de la nationalité bulgare, et essaya même de l'instrumentaliser dans sa politique active par rapport à la Yougoslavie en proclamant le Pirin comme la seule partie de la Macédoine où la " population macédonienne " se sentirait " libre ${ }^{26}$. De plus, sous la pression soviétique, dès 1955, la Bulgarie entreprit un nouveau rapprochement avec son voisin de l'ouest et on peut tenter d'éclairer les données du deuxième recensement à la lumière de cette conjoncture politique et idéologique. Aussi, à en croire les sources du Parti, le succès de la politique de "macédonisation " des années 1950 est aussi la conséquence d'une certaine confusion au niveau local entre deux registres d'identification : d'un côté " Macédonien/ne » dans un sens régional, équivalent égal à " Bulgare macédonien/ne ", et d'un autre - " Macédonien/ne eth-

\footnotetext{
23 Angelov (Veselin), op.cit., p. 289 ; Germanov (Stojan), « Načaloto na preocenka na bălgarskata politika po makedonskija văpros 1948-1963 g. ", Makedonski pregled, (1), 2003, p. 109.

24 Voir CPA, F. 1, OP. 6, A.E. 3023, L. 53-54, 57-59-suggestion de l'Administration Centrale de Statistique auprès du Conseil des ministres (7166), 25/08/1956, faisant partie de la Résolution du Politbureau du Comité central du PCB du o4/10/1956. Voir aussi les directives du Comité départemental de Blagoevgrad concernant le recensement - Archives départementales du Parti (Okräžen partien arhiv), ci-après OPA, F. 2, OP. 1, A.E. 164, L. 6 de 03/11/1956 : "Que chacun définisse sa nationalité d'une manière libre. Pas de violence ".

25 OPA, F. 2, OP. 1, A.E. 164 , L. 3.

26 Voir les « Directives pour les comités et les organisations du Parti dans la région du Pirin " du 20 décembre 1951 - CPA, F. 1, OP. 64, A.E. 160.
} 
nique ", distinct de " Bulgare ", "Serbe " ou " Grec/que "27. Déjà en 1948, un des secrétaires locaux de Parti constata que "La population ne fait pas de distinction entre Bulgare et Macédonien. J'ai posé à plusieurs personnes la question de savoir ce qu'elles sont [de nationalité], et elles me répondent : "Macédonien

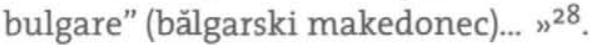

En revanche, au cours du troisième recensement de l'époque communiste, en 1965 , la " Question macédonienne " en Bulgarie se présentait radicalement différemment : le Parti communiste et l'État bulgare ne reconnaissaient plus ni la langue ni la nationalité macédonienne. Dès 1956, l'emprise progressive du pouvoir par des nouveaux cadres du Parti dirigés par Todor Živkov et partisans d'une politique assimilatrice forte vis-à-vis des minorités nationales - marqua la fin de la reconnaissance non seulement d'une minorité macédonienne mais, en général, d'une identité ethnique macédonienne distincte de l'identité bulgare ${ }^{29}$. Cette position fut officiellement formulée lors d'un Plénum du Comité central en mars $1963^{30}$. Devant les membres du Parti à Blagoevgrad, le secrétaire du Comité central, Mitko Grigorov, n'hésita pas à reprocher cette " macédonisation " du Pirin à Georgi Dimitrov, décédé en 1949, et officiellement glorifié comme " dirigeant et maître du peuple bulgare " $3^{31}$. Au recensement de 1965, le nombre de Macédoniens se réduit brusquement : 9632 personnes dont 1437 dans la région du Pirin c'est-à-dire moins de $0,5 \%$ de la population totale de la région ${ }^{32}$. Seuls 462 Macédoniens déclarèrent comme

27 Le sens régional du " Macédonien " dans le Pirin en 1956 est accentué dans les rapports des fonctionnaires du Parti - voir CPA, F. 1, OP. 13, A.E. 112, L. 2, L. 23 ; CPA, F. 1, OP. 6, A.E. 3023, L. 53-59.

${ }^{28}$ CPA, F. 1, OP. 13, A.E. 112, L. 2, L. 23. D'après certaines sources, les gens du Pirin référeraient souvent la dénomination "Macédonien " aux membres de l'Organisation révolutionnaire macédonienne (d'option ethnique bulgare) VMRO de la période de l'Entre-deux-guerres. Une partie de la population n'en garderait pas de bons souvenirs et aurait des stéréotypes envers l'identification " Macédonien n même - voir CPA, F. 1, OP. 5 , A.E. 21, L. 13.

29 Cf. la monographie de Troebst (Stefan), Die bulgarisch-jugoslawische Kontroverse um Makedonien 1967-1982, München / Oldenburg : Südost-Institut, 1983, qui demeure jusqu'à maintenant un guide irremplaçable dans les intrications de la politique bulgare et yougoslave sur la "Question macédonienne " pendant le communisme.

30 Voir les discussions lors du Plénum du 11-12 mars 1963 publiées dans le recueil de documents : BKP, Komiternät $i$ makedonskijat väpros (1917-1946), II, Sofia : GUA pri MS, 1999, pp. 1286-1294, et chez Germanov (Stojan), art.cit., pp. 113-136. Voir aussi l'analyse critique de Angelov (Veselin), " Za roljata i mjastoto na Martenskija plenum na CK na BKP ot $1963 \mathrm{~g}$. za formirane i proveždane na bălgarskata politika po makedonskija văpros ", Minalo, (1), 2001 / 1.

31 OPA, F. 2, OP. 4, A.E. 1, L. 22.

32 Rezultati ot prebrojavaneto na naselenieto na 1.XII.1965. Okrăg Blagoevgrad, Tom I, 1, Sofia : CSU, 1967, pp. 11-12, p. 118, p. 125. Dans la littérature historiographique sur ce sujet, on se réfère presque sans exceptions aux figures erronées de 8750 Macédoniens au total contre 1500 dans le Pirin - cf. pars pro toto, Tintoski (Stevan), Makedoncite vo Bugarija, Skopje : Studentski zbor, 1987, p. 99. 
langue de communication " autre langue " - au lieu du bulgare - dont seulement 8 personnes dans le département de Blagoevgrad. La presse et l'historiographie de Skopje dénoncèrent, bien sûr, les truquages et le " génocide statistique "33. Mais la piste statistique pour connaître les dimensions de l'identité ethnique macédonienne dans le Pirin semble être plus embrouillée que cela.

Le recensement de 1965 fut précédé par une campagne de renouvellement des documents d'identité (passeports de circulation à l'intérieur du pays et autres documents), campagne destinée à corriger les origines officiellement " macédoniennes " de la majorité de la population du Pirin. Jusqu'à présent, les données documentaires disponibles n'ont montré aucune infraction ou irrégularité durant cette campagne ou le recensement qui la suivit. La direction centrale du Parti et le bureau du Comité départemental à Blagoevgrad préconisèrent d'éviter des " déviations " (izvraštenija) au cours de la procédure de " changement de passeports" (pasportizacija) et du recensement, tout en respectant l'auto-identification ethnique de la population du Pirin 34 . Le Secrétaire du Comité central du PCB conseilla aux autorités locales de prendre en compte la volonté de chaque personne : " ceux qui ont mûri (uzreli) comme Bulgares, qu'on les enregistre comme "Bulgares", ceux qui sont Macédoniens qu'ils restent "Macédoniens" "35. Le Politbureau du Parti garantit aux citoyens une " liberté complète " (pălna dobrovolnost) quant au choix de nationalité ${ }^{36}$.

D'après les sources du Secrétariat du Comité central, avant même le processus de changement des cartes d'identité, en 1963,42,4\% des nouveau-nés de la région étaient enregistrés comme "Bulgares " contre $24,4 \%$ en 196037 . En 1965, la direction départementale du Parti constata que, pendant les quatre premiers mois de l'année, $85 \%$ des gens optèrent dans leurs passeports pour l'origine " Bulgare ", contre $11 \%$ de " Macédoniens " $^{8}$. D'après le premier secrétaire du Comité départemental du Parti, la population accepta la possibilité de s'enregistrer comme "Bulgare " avec " satisfaction " (zadovolstvo) et le fit de son propre gré (dobrovolno) 39 . De cette façon, juste à la veille du recensement, les gens du Pirin reçurent de nouveaux documents où leur " nationalité " (narodnost) était désormais " bulgare ". Mais même sur la base

\section{Tintoski (Stevan), op.cit., p. 113.}

34 OPA, F. 2, OP. 5, A.E. 6, L. 19 de mai 1965.

35 OPA, F. 2, OP. 4, A.E. 1, L. 23. Voir aussi Ibid., L. 72 : " On ne doit pas user de la violence (nasilie) sur qui que ce soit dans la déclaration de sa nationalité " "

${ }^{36}$ CPA, F. 1, OP. 6, A.E. 5426 , L. 10.

37 CPA, F. 1, OP. 8 , A.E. 6561 , L. 34 .

38 OPA, F. 2, OP. 5, A.E. 6, L. 19. Le pourcentage de " Macédoniens " resta élevé, voire majoritaire, à Krupnik, Kolarovo, Selište, dans l'aire de Ogražden, etc.

39 CPA, F. 1, OP. 8, A.E. 7981, L. 6. 
des directives officielles disponibles, l'écart énorme entre les données de 1956 et celles de 1965 semble inexplicable.

Les militants macédoniens en Bulgarie de même que les historiens en République de Macédoine relatent des cas de " résistance " à cette suppression officielle de la nationalité macédonienne sur les cartes d'identité40. D'après leurs sources, si certains choisirent effectivement une identification nationale bulgare, d'autres virent cette identification inscrite d'office sur leur nouveau passeport. Dans le Pirin, selon le parti politique OMO "Ilinden ", les employés d'État ne respectaient pas la volonté des personnes de la région insistant pour être enregistrées dans leurs nouveaux passeports comme Macédoniennes. Mais, en même temps, à l'intérieur du pays - à Sofia, Plovdiv ou Varna - il aurait été beaucoup plus facile de garder la nationalité macédonienne ${ }^{41}$. Ces souvenirs communiqués par des activistes macédoniens de Bulgarie semblent être confirmés par la tendance relativement stable du nombre de Macédoniens dans les autres régions du pays - entre 8 ooo et 9000 personnes en 1946,1956 et 1965, faisant contraste avec la disparition de plus de 177000 Macédoniens antérieurement recensés dans le Pirin. À partir de ces données, on peut conclure que l'État bulgare était relativement plus intransigeant envers l'auto-identification macédonienne dans cette région qui faisait l'objet de controverses aiguës avec la Yougoslavie. En mars 1963, Živkov, lui-même, décréta ex cathedra que, dans le Pirin, " il n'y a aucune nationalité (nacionalnost) macédonienne $" 4^{2}$. Ainsi, les mécanismes d'homogénéisation nationale de cette époque, focalisés sur la région du Pirin, laissèrent-ils échapper à la nouvelle ligne politique les Macédoniens des territoires éloignés de la frontière avec la Yougoslavie.

Il faut également souligner qu'au cours du changement des documents d'identité et du recensement, une campagne vigoureuse d' "éclaircissement " (razjasnitelna rabota) de la "Question macédonienne " fut lancée dans le département de Blagoevgrad par la direction centrale et départementale du Parti43. Le récit historiographique traditionnel sur le " caractère bulgare " de la Macédoine fut instrumentalisé par le sommet politique et académique bulgare pour " éduquer " la population de la Macédoine du Pirin. Cette campagne exerça, sans doute, un certain impact sur la manière dont les locaux déclarè-

40 Cf. Tintoski (Stevan), op.cit., p. 100.

${ }^{41}$ Information orale de Dimităr Ivanov, ex-membre de l'organisation « Militants pour la réunification de la Macédoine " (Ratnici za obedinenieto na Makedonija) et activiste macédonien habitant à Šumen, Bulgarie du nord-est.

$4^{2}$ BKP, Komiternät i makedonskijat văpros (1917-1946), (op.cit.), p. 1292.

43 Voir les directives officielles - CPA, F. 1, OP. 6, A.E. 5426, L. 10 du 07/04/1964; CPA, F.1, OP. 8, A.E. 68 O2, L. 3-46 de janvier 1965 ;OPA, F. 2, OP. 5, A.E. 21 d'avril 1965. Voir aussi OPA, F. 2, OP. 5, A.E. 3, L. 36-40 de février 1965. 
rent leur nationalité en 1965 : selon le " principe de base " (izhodna postanovka) officiellement annoncé lors de la campagne d' " éclaircissement ", " la nationalité (narodnost) macédonienne n'a [jamais] existé ", la population de la région étaient d'" origine bulgare ", la Yougoslavie "falsifiait " les "faits historiques " 44 . De cette façon, la population du département de Blagoevgrad fut " avertie " de l'option ethnique agrée par les autorités.

Aussi, faut-il ajouter la spécificité de la méthode de recueil de données sur l'origine ethnique. Déjà lors du recensement de 1956, le rôle des agents recenseurs fut progressivement renforcé et, malgré les directives officielles préconisant de respecter l'auto-identification des personnes recensées, les employés d'État tendaient à enregistrer plutôt les données sur la nationalité figurant sur les cartes d'identité45. Or, si en 1956 la majorité de la population du Pirin était officiellement " macédonienne ", en 1965, c'est une origine ethnique " bulgare " qui était indiquée dans ses passeports. C'est peut-être la raison pour laquelle, dans les récits des activistes macédoniens aujourd'hui, l' "effacement " de la nationalité macédonienne est daté de la campagne de renouvellement des passeports et non du recensement. Tous ces éléments confirment le caractère artificiel et subjectif des statistiques de 1965 sur la nationalité. Mais la contestation des données sur les "Macédoniens ethniques " en Bulgarie à partir de 1965 pourrait du même coup mettre en doute la validité des recensements précédents, particulièrement celui de 1956 qui fut effectué selon la même méthode. D'une façon sélective, les spécialistes macédoniens ne doutent jamais de la véracité de ces derniers, alors que leurs collègues bulgares tendent à remettre en question même l'existence des quelques milliers de Macédoniens recensés en $1965^{46}$. Aujourd'hui, les estimations exagérées qui avancent le chiffre de 200000 "Macédoniens ethniques " en Bulgarie se fondent notamment sur les données des deux premiers recensements effectués sous le régime communiste et estimés comme seuls " véridiques".

Le quatrième recensement de l'époque communiste - celui de 1975 - ne dévoila pas au public les données sur les groupes ethniques alors même qu'ils furent recensés 47 . Enfin, le dernier recensement de l'époque - celui de 1985 - n'at-

44 OPA, F. 2, OP. 4 , A.E. 1, L. 65-72.

45 Voir Arkadiev (Dimităr), « Izučavane na etničeskija săstav pri prebrojavanijata na naselenieto v Bălgarija ", Naselenie, (6), 1992, p. 53.

46 Angelov (Veselin), art.cit., p. 91.

47 Prebrojavane na naselenieto i żilišstnija fond käm 2.XII.1975. Demografska i ikonomičeska harakteristika na naselenieto $v N R B$, KESSI pri MS, 1977 : sur la p. 11, on voit le carton de recensement qui prévoit la case nationalité (narodnost) avec l'exigence de marquer la nationalité des " personnes qui ne sont pas Bulgares ". Le recensement attesta la présence de Macédoniens mais leur nombre reste inconnu - cf. Arkadiev (Dimităr), art.cit., p. 55. 
testa pas de nationalités. Après la chute du communisme, les données du recensement de 1992 sur les Macédoniens restèrent non-publiées mais furent annoncées par un conseiller du Président de la république qui avança le nombre de 10803 Macédoniens dont 3109 déclarant le macédonien comme langue maternelle ${ }^{8}$. En revanche, les dernières statistiques sur les " groupes ethniques " (etničeski grupi) en Bulgarie - celles de 2001 - sont bien disponibles49. Pourtant, au lieu de présenter la " large minorité macédonienne " avancée par un bon nombre de militants d'ONG, de journalistes, de politiciens et de scientifiques, ces derniers résultats ne font état que de 5071 personnes en Bulgarie se déclarant comme appartenant au " groupe ethnique macédonien " dont la majorité sont effectivement habitants de la région du Pirin (3 117 personnes).

\section{Les habitants de « nationalité macédonienne 》}

\begin{tabular}{|c|c|c|c|c|c|c|c|}
\hline & 1946 & 1956 & 1965 & 1975 & 1985 & 1992 & 2001 \\
\hline $\begin{array}{l}\text { MACÉDONIENS } \\
\text { EN BULGARIE } \\
\text { - TOTAL }\end{array}$ & 169544 & 187789 & 9632 & $\begin{array}{c}? \text { (recensé } \\
\text { mais non } \\
\text { publié) }\end{array}$ & $\begin{array}{c}? \\
\text { (non } \\
\text { recensé) }\end{array}$ & $\begin{array}{l}10803 \\
\text { (non } \\
\text { publié) }\end{array}$ & 5071 \\
\hline $\begin{array}{l}\text { MACÉDONIENS } \\
\text { DANS LA RÉGION } \\
\text { DU PIRIN }\end{array}$ & 160641 & 178862 & 1437 & $\begin{array}{c}\text { ? (recensé } \\
\text { mais non } \\
\text { publié) }\end{array}$ & $\begin{array}{c}\text { ? } \\
\text { (non } \\
\text { recensé) }\end{array}$ & $\begin{array}{c}? \\
\text { (non } \\
\text { publié) }\end{array}$ & 3117 \\
\hline
\end{tabular}

Tableau 2 : Données statistiques d'après les sources disponibles (sauf pour 1946 et 1992). " Région du Pirin " = "Région (oblast) de Gorna Džumaja " (1946), " Département (okräg) de Blagoevgrad " (1956, $1965,1975,1985)$, " Région (region) de Blagoevgrad " dans le cadre de la " Région (oblast) de Sofia " (1992) et " Région (oblast) de Blagoevgrad " 2001).

D'un côté, on peut donc légitimement s'interroger sur les méthodes formelles de recensement de la nationalité en 1946, 1956 mais aussi en 1965 qui dévoilaient, dissimulaient ou construisaient les auto-identifications individuelles des gens du Pirin. La référence non-critique à des données de ce genre peut passer pour un acte relevant de certains agendas politiques, qu'ils soient ceux d'institutions d'État ou d'organisations non gouvernementales, plutôt que pour une " analyse scientifique ". Sur la base des données disponibles, on peut, de l'autre côté, avancer l'hypothèse que les catégories d'identification de la majorité des personnes en Macédoine du Pirin changeaient chaque fois en

$4^{8}$ Voir Ivanov (Mihail), art.cit., qui avait alors la fonction de conseiller sur les affaires ethniques du président Želev. Les données publiées enregistrèrent, semble-t-il, les Macédoniens sous la rubrique "Autres ". Voir Prebrojavane na naselenieto $i$ žilištnija fond kăm 4 dekemvri 1992. Socialno-demografski harakteristiki. Region Blagoevgrad, Sofia : NSI, 1996, pp. 147-149. Il y aurait 10769 " Autres n dans la région de Blagoevgrad (p. 147).

49 Aussi en ligne sur http://www.nsi.bg/Census/Census.htm. 
fonction d'une conjoncture politique et culturelle laquelle exerçait en premier lieu une violence symbolique ${ }^{50}$ incontestable sur les habitants de la région. La politique officielle bulgare a, au départ, encouragé l'option identitaire macédonienne, puis, à partir de la fin des années 50, opté en faveur de l'identité nationale bulgare. Dans le Pirin, ces oscillations idéologiques donnaient naissance à des choix identitaires différents qui allaient de la conformité totale jusqu'à la résistance, souvent suivie d'une répression réelle - éviction du Parti, licenciement, déportation, emprisonnement. Les données d'archives qui suivent devraient, au moins partiellement, préciser ce climat idéologique en Macédoine du Pirin de cette époque.

\section{LE « NATIONALISME » ET L'« ÉDUCATION PATRIOTIQUE 》 DANS LE PIRIN}

Résultat des Guerres balkaniques et de la Première Guerre mondiale, l'intégration de la Macédoine du Pirin au sein de l'État bulgare ne rencontra pas de résistance relevant de motifs ethno-nationaux macédoniens ${ }^{51}$. L'exode progressif de la population qui s'identifiait comme grecque et d'une partie de la population musulmane fut compensé par l'afflux de réfugiés des deux autres parties de la Macédoine, surtout celle de l'Égée, lesquels contribuèrent à l'" homogénéité " de la région. Si du point de vue bulgare celle-ci est " purement bulgare ", dans l'imaginaire national macédonien d'aujourd'hui, le Pirin représente la partie la plus " ethniquement pure " des trois parties de la Macédoine géographique ${ }^{52}$. Il faudrait cependant remarquer la présence d'une importante minorité musulmane bulgarophone (pomaque) dans la partie est de la région.

$\mathrm{Au}$ cours de l'entre-deux-guerres, le chef de l'aile dominante au sein de l'Organisation révolutionnaire macédonienne VMRO, Ivan (Vančo) Mihajlov,

50 D'après Bourdieu : « La violence symbolique, c'est cette violence qui extorque des soumissions qui ne sont même pas perçues comme telles en s'appuyant sur des "attentes collectives", des croyances socialement inculquées... " (Bourdieu (Pierre), Raisons pratiques. Sur la théorie de l'action, Paris : Seuil, 1994, p. 188).

${ }^{51}$ Mis à part certaines interprétations historiographiques revendiquant un « caractère national macédonien " du mouvement partisan communiste dans le Pirin lesquelles sont en train d'être révisées par les historiens macédoniens mêmes (voir Katardžiev (Ivan), Makedonija sto godini po Ilindenskoto vostanie, Skopje : Kultura, 2003, p. 280). L'historien macédonien Katardžiev est lui-même originaire de la Macédoine du Pirin et faisait partie de ces étudiants de la région qui, au cours de l'" autonomie culturelle " et grâce à des accords bilatéraux, partirent poursuivre leurs études à Skopje et préférèrent y rester.

${ }^{2}$ Cf. Kiselinovski (Stojan), Etničkite promeni vo Makedonija 1913-1995, Skopje : INI, 2000, p. 55. 
transforma la Macédoine du Pirin en une région autonome, une sorte d'“ État dans l'État ", qui deviendra une base pour pour la propagation de l'irrédentisme bulgare dans les autres parties de la Macédoine ${ }^{53}$. Or, la " macédonisation " du Pirin entre 1944 et 1948 a dû faire face à l'opposition anticommuniste et surtout à celle des adeptes de Mihajlov - les " mihajlovistes "-entre-temps réprimés et marginalisés par le pouvoir. Ces derniers incarnaient un certain type de " nationalisme politique " macédonien fondé sur la thèse de l'ethnicité bulgare de la population slave de Macédoine : l'idéologie mihajloviste postule l'existence d'une communauté particulière, nommée "peuple macédonien " (makedonski narod) et pensée comme une alliance de différentes ethnies dont, en première place, " les Bulgares macédoniens ". La seule résistance au " régime bulgare " dans la région pendant cette période est portée par des groupes armés dits mihajlovistes mais elle est plus le résultat d'une attitude anticommuniste, incitée aussi par la collectivisation des propriétés agricoles, que d'un mouvement d'affirmation d'une identité nationale macédonienne distincte ${ }^{54}$.

Cependant, le conflit avec le régime de la Yougoslavie, suivi par le dénonciation officielle de Tito comme " valet de l'impérialisme américain ", provoqua, dans un premier temps, une certaine mutation idéologique au sein des milieux mihajlovistes partisans d'une " grande Macédoine " indépendante mais sous une tutelle britannique et américaine. D'abord, adversaires de la politique de " macédonisation " stigmatisée comme " pro-serbe ", en 1948-1949, les mihajlovistes s'emparèrent - d'après les sources du Parti - de la dénomination "Macédonien ", cette fois-ci perçue comme un enjeu symbolique contre la nouvelle ligne officielle des communistes. Un groupe anticommuniste nommé "Organisation illégale de libération de la Macédoine " (NOMO) fut créé par des mihajlovistes de Bansko et du district de Nevrokop55. Ainsi, le " nationalisme politique " bulgaro-macédonien de ces derniers, condamné d'abord comme

53 En 1934, un coup d'État qui met au pouvoir un nouveau gouvernement pro-yougoslave et anti-mihajloviste met aussi fin à cette " autonomie n du Pirin mais sans pouvoir complètement paralyser l'activité politique de Mihajlov et de ses adeptes. La dernière étude documentaire bulgare sur le quasi-État mihajloviste dans le Pirin est celle de Tjulekov (Dimităr), Obrečeno rodoljubie. VMRO v Pirinsko 1919-1934, Blagoevgrad : Universitetsko izdatelstvo, 2001, accessible aussi en ligne sur http://knigite.abv.bg/dt/index.html .

54 Voir les recueils de documents sur le groupe le plus important de ce genre - la " bande n de Gerasim Todorov : Angelov (Veselin), Otličen bălgarin s imeto Gerasim, Sofia, 2001 ; Gorjanite. Sbornik dokumenti 1944-1949, GUA pri MS : Sofia, 2001, pp. 260-353. Sur le sort des mihajlovistes en Bulgarie communiste Nedeva (Ivanka), Kajžev (Naum), " IMRO Groupings in Bulgaria after the Second World War ", in Pettifer (James), éd., The New Macedonian Question, Hampshire / London : Macmillan Press, 1999.

55 Angelov (Veselin), Gorjanite (op.cit.), pp. 110-113. Les mihajlovistes diffusèrent des proclamations anticommunistes pour une "Macédoine autonome "-voir un appel de ce genre, destiné aux " peuples macédoniens de la Macédoine du Pirin n dans Archives du Ministère de l'Intérieur (Arhiv na Ministerstvoto na vătrešnite raboti) AMVR, F. 2, OP. 1, A.E. 80, L. 3. 
" chauvinisme grand-bulgare ", fut-il, par la suite, fustigé par le Parti comme étant de nature " pro-yougoslave ${ }^{56}$. En avril 1950, à Gorna Džumaja (Blagoevgrad), douze personnes, en partie " ex-mihajlovistes ", furent accusées de séparatisme en tant que " agents de Tito " et poursuivies en justice57. Toujours en 1950, à Bansko, le régime du Parti communiste donna naissance à des sentiments macédoniens anti-bulgares exprimés par le slogan "Vive la Macédoine indépendante - dehors les occupants bulgares " ${ }^{58}$. Aujourd'hui, les militants macédoniens en Bulgarie appartenant à des organisations comme $\mathrm{OMO}$ “ Ilinden " se revendiquent de cette lignée 59 .

Or, il serait très hasardeux de tracer une continuité directe entre la tradition mihajloviste dans le Pirin et les cas d'ethno-nationalisme macédonien attestés dans la région à partir de 1948. À présent, les nationalistes macédoniens du Pirin se réfèrent exclusivement à la mémoire de l'aile dite de gauche de l'ancienne Organisation révolutionnaire macédonienne, tout en étant hostiles à l'égard de la "droite fasciste " de Mihajlov. À présent, chaque année l'organisation OMO "Ilinden " commémore deux anniversaires importants : la mort de Jane Sandanski (22 avril 1915) - leader de l'aile gauche, avant les Guerres balkaniques très actif dans le Pirin, et les assassinats de gauchistes et de communistes macédoniens ordonnés par Mihajlov (12 septembre 1924). Cette date est marquée par les " macédonistes " en Bulgarie comme " jour du génocide bulgare sur le peuple macédonien " (sic). Malgré son lien idéologique possible au niveau local avec le " camp opposé " de l'indépendantisme mihajloviste, ce n'est que l'héritage " autonomiste " de la " gauche " de Sandanski qui laissa à long terme son empreinte sur les manifestations d'une identité macédonienne non-bulgare dans la région sous le communisme et après.

Le Parti lança, en revanche, une activité de propagande qualifiée d'" éducation de classe, patriotique et internationaliste " ou simplement d'" éducation patriotique "(patriotično văzpitanie) ayant au départ l'objectif de cultiver, dans le dé-

56 Voir CPA, F.1, OP. 13 , A.E. 112, L. 9, L. 23 ;CPA, F. 1, OP. 12, A.E. 404, L. 7-8 ;CPA, F. 1, OP. 12, A.E. 452, L. 16, L. 23 ; CPA, F. 1, OP. 12, A.E. 465 , L. 18 : les mihajlovistes montreraient de l'intérêt envers la Yougoslavie et Tito, seraient les seuls lecteurs du quotidien de Skopje Nova Makedonija, s'afficheraient comme " Macédoniens " etc. À Nevrokop (Goce Delčev), les mihajlovistes établiraient contact avec le libraire macédonien de Skopje - OPA, F. 5, OP. 1, A.E. 5, L. 88. Des aspects ethno-nationaux macédoniens au sein de l'idéologie mihajloviste sont attestés par les historiens macédoniens (voir Katardžiev (Ivan), Katardžiev, op.cit., p. 224) mais furent rejetés par Mihajlov et sont toujours fort contestées - voir Tjulekov (Dimităr), op.cit., http://knigite.abv.bg/dt/dt3_4.html, note 267 .

57 Le groupe de Nikola Rolev (Koče) - OPA, F. 2, OP. 1, A.E. 529, L. 137. Voir aussi Gorjanite (op.cit.), p. 566-572. $5^{8}$ Le cas de Nikola Kolčagov - OPA, F. 2, OP. 1, A.E. 528, L. 143 de 1950-1051.

59 Selon les données de OMO “llinden”, cinq personnes du groupe de Rolev furent condamnées à mort, alors que les autres passèrent de longues années en prison : cf. Poulton (Hugh), op.cit., p. 149. Un ex-membre de la bande de Todorov, - Krum Monev, présenta l'activité de celle-ci comme relevant d'une idéologie ethno-nationale macédonienne : voir ses mémoires samizdat (Monev (Krum), Makedonija. Mojata opora, s.e., 1992). 
partement de Blagoevgrad, une macédonité anti-titiste et de caractère linguistique bulgare ${ }^{6 \circ}$. Au début des années 1950, le Parti jugea toujours inacceptable le refus d'une partie de la population du Pirin de se reconnaître de nationalité macédonienne - des cas de ce type sont, par exemple, rapportés de la ville de Sandanski (anciennement Sveti Vrač) ${ }^{61}$. C'est pourquoi les directives officielles préconisèrent à la fois une " lutte " contre l'influence yougoslave et le " chauvinisme grandbulgare ", les " titistes " et les " mihajlovistes " étant stigmatisés à pied égal en tant que " ennemis du peuple " ${ }^{62}$. Or, au cours des années 1950, l'identité macédonienne " pro-bulgare " plébiscitée par le Parti se réduisit exclusivement à la promotion du folklore local comme attesté par la création d'ensembles de chants et de danses populaires macédoniens dont le plus important - Pirin - fut fondé en 1954.

À la fin des années 1950, le tournant de la politique officielle s'affirma-la " macédonisation " fut définitivement abandonnée ce qui suscita l'élaboration d'une nouvelle position d'État sur la "Question macédonienne ". En 1957, plusieurs fonctionnaires du Parti exprimaient leur inquiétude qu'une " bonne partie " de la population du Pirin " se sent macédonienne "63. La même année, la direction du Comité départemental de Blagoevgrad exclut du Parti plusieurs séparatistes du district de Petric ${ }^{64}$. L'abrogation progressive de la nationalité macédonienne en Bulgarie provoqua des résistances individuelles ou organisées, publiquement condamnées par le pouvoir comme " actes nationalistes " (nacionalističeski projavi). Le " patriotisme " dans sa version marxiste-léniniste se veut " populaire " et " internationaliste " pour s'opposer au " nationalisme " stigmatisé comme un phénomène " bourgeois " : en utilisant ce concept, le Parti bannit les actes de nationalisme macédonien dans le Pirin et ailleurs en Bulgarie. Ces derniers s'exprimaient par la création de groupes pour " la libération et la réunification de la Macédoine ", par des graffiti et des slogans, par la diffusion de pétitions, par des revendications verbales, etc.

Par exemple, en 1954, des étudiants de la région du Pirin insistèrent sur l'enseignement en langue macédonienne littéraire, officiellement rejetée par le pouvoir comme une variante linguistique " serbisée " : les étudiants étaient particulièrement mécontents que certains poètes originaires de la Macédoine comme Smirnenski et Vapcarov figurent dans le programme scolaire comme

60 Voir CPA, F. 1, OP. 15 , A.E. 569 , L. 7 de 1953.

${ }^{61}$ CPA, F. 1 , OP. 15 , A.E. 405 , L. 4 ; OPA, F. 2, OP. 1, A.E. 524 , L. 114.

62 Voir pars pro toto CPA, F. 1, OP. 6, A.E. 526 ; OPA, F. 2, OP. 1, A.E. 5, L. 148 ; OPA, F. 2, OP. 1, A.E. 526, L. $34-35$.

63 CPA, F. 1, OP. 6, A.E. 3417 , L. 19 de $24 / 10 / 1957$.

64 OPA, F. 2, OP. 1, A.E. 166, L. 102 de janvier 1957 -il s'agit de personnes des villages de Kolarovo, Samuilovo etc. Un parmi eux - Andon Zajkov - aurait élaboré le statut de l'organisation nommée OOM dont l'objectif était la " réunification de la Macédoine ". Voir aussi OPA, F. 2, OP. 5, A.E. 11, L. 71. 
" poètes bulgares "65. Des instituteurs du district de Sandanski dénoncèrent l'absence de " culture de forme macédonienne "66. Une enquête officielle dans un lycée de Sandanski constata la perpétration d' "actes nationalistes ". En 1961, un rapport du Ministère de l'Intérieur destiné au Comité central du Parti fit état des dimensions des " attitudes nationalistes " dans le département de Blagoevgrad : pendant les deux années précédentes furent remarqués plusieurs groupes de petite taille (jusqu'à neuf personnes, surtout des adolescents) qui diffusaient l'idée d'une unification de la région avec la Macédoine yougoslave $^{67}$. Le meurtre de deux adolescents du village de Ključ, qui essayaient de passer clandestinement en Grèce, donna également lieu à des slogans anti-bulgares en plusieurs endroits près de Petrič 68 .

En 1964, sur la base d'un rapport du premier secrétaire départemental du Parti, Krăstju Tričkov, le Secrétariat de Comité central amorça une vaste série de mesures pour l'" éducation patriotique "dans le Pirin : la population du Pirin devait dorénavant faire partie de la nationalité bulgare ${ }^{69}$. Des directives de ce genre furent appliquées de manière brutale à la population musulmane de la région, obligée d'abandonner ses noms personnels d'origine turco-arabopersane pour acquérir des noms bulgares ${ }^{70}$. Quant à la "Question macédonienne ", toutes les structures du Parti et des autres organisations politiques officielles ainsi que les institutions culturelles régionales et nationales furent engagées dans ce processus ayant pour objectif de prévenir les " actes nationalistes " qui s'activèrent en 1964-1965. Seulement entre le $1^{\text {er }}$ janvier et le 20 août 1965 on trouva plus de 17 cas de diffusion de slogans et de proclamations séparatistes ${ }^{71}$. La jeunesse et, particulièrement celle des lycées de Petrič et de Sandanski fit l'objet d'une attention spéciale ${ }^{72}$. Cette activité était indispensable pour la " bonne conduite " de la campagne de renouvellement des cartes d'identité et du recensement en décembre 1965 lequel ne tarda pas à faire preuve du " succès " du Parti. Mais jusqu'à la fin des années 1960, la " lutte "

$65 \mathrm{ll}$ s'agit d'un cas à l'Institut pédagogique à Stanke Dimitrov (Dupnica), possiblement relié à des revendications de ce genre qui eurent lieu en même temps à Sofia - CPA, F.1, OP. 16, A.E. 13, L. 1 de 01/04/1954. 66 CPA, Ibid., L. 6-7.

67 CPA, F. 1, OP. 24, A.E. 272, L. 2-6 : seulement au cours de 1960, 90 " intentions (namerenija) de haute trahison " furent rapportées, mais pas forcément relevant de nationalisme macédonien. Voir aussi CPA, F. 1, OP. 12, A.E. 1127 , L. 16 de 1961.

68 OPA, F. 2, OP. 5, A.E. 9 et A.E. 24 de juillet 1965 : il s'agit des adolescents Georgi Gecov et Pande Pandeliev.

69 Voir CPA, F. 1, OP. 8, A.E. 6561 , L. 3-36 et OPA, F. 2, OP. 6, A.E. 4.

$70 \mathrm{Ce}$ qui provoqua un véritable soulèvement populaire dans les villages musulmans de Ribnevo, Kornica, Breznica etc., où, pour " apaiser " la situation, la direction du Parti et de l'État envoya aussi des divisions de l'armée. Pour plus de détails - voir CPA, F. 1, OP. 6, A.E. 5454.

71 OPA, F. 2, OP. 5, A.E. 11, L. 70. Il s'agit de gens de l'aire de Blagoevgrad, Ograżden, Gara Pirin, Petrič, etc.

72 OPA, F. 2, OP. 5, A.E. 23 de 1965 : des " actes nationalistes n commis par lycéens sont rapportés pour 1964. Voir aussi OPA, F. 2, OP. 4, A.E. 1, L. 4-5. 
avec le nationalisme macédonien dans le Pirin continua activement, relancée en 1967-1968 lorsque les controverses politico-historiographiques avec la Macédoine yougoslave devinrent extrêmement violentes.

À ce moment, le Comité départemental " constata " une réactivation du " nationalisme à la Skopje ", surtout dans le district de Petrič 73 . En novembre 1967 , le Secrétariat du Comité central du PCB consacra une nouvelle séance spéciale à l'" éducation de classe et patriotique de la classe ouvrière " (trudeštite se) de la région après avoir reçu un rapport alarmant de la part du premier secrétaire départemental : entre 1965 et 1967,15 groupes " nationalistes " auraient été dépistés dans le Pirin, avec environ 90 membres au total, partisans d'une " Macédoine libre et indépendante "74. En 1967, dix adolescents furent accusés d'avoir diffusé des proclamations à " contenu nationaliste " tandis que, lors d'un concert de folklore à Mikrevo, des jeunes du village refusèrent d'écouter des chansons populaires non-macédoniennes 75 . La direction du Parti répondit avec une nouvelle offensive visant à cultiver dans la population du Pirin " le sentiment de fierté qu'elle fut toujours et continue à être une partie intégrante du peuple bulgare $»^{76}$. Le Politbureau accéléra la nouvelle ligne politique avec une résolution sur la "Question macédonienne " et le Secrétariat précisa les " mesures " pour sa mise en place dont une partie concernait en particulier le département de Blagoevgrad 77 . Des écrivains, des historiens et d'autres spécialistes bulgares de la "Question macédonienne " (souvent eux-mêmes originaires de Macédoine) furent invités à donner leur contribution à l'" éducation patriotique " des locaux sous la forme de conférences et de réunions. Néanmoins, en 1968, le nombre de groupes " nationalistes " et de leurs membres était en augmentation. D'après les données du chef de la section à Blagoevgrad du Comité pour la Sécurité d'État (KDS), entre 1965 et 1968, ce seraient 28 groupes avec 136 membres au total plus 22 personnes qui n'appartiendraient à aucun groupe. En juillet 1968, dans le village de Musomišta, près de la ville de Goce Delčev, 80 personnes " macédonisantes " (makedonstvašti) se réunirent ${ }^{7}$. Entre 1966 et 1970, à Katunci et à Petrovo, ce sont cinq groupes " nationalistes " qui furent démantelés79.

73 OPA, F. 2, OP. 7, A.E. 12 , L. $43-47$.

74 CPA, F. 1, OP. 8, A.E. 7981, L. 6-9. Des cas « nationalistes " furent constatés même dans l'actif du Parti à Petrič, dans l'aire de Ogražden, à Gara Pirin et à Kolarovo.

75 OPA, F. 2, OP. 6, A.E. 14A, L. 17-22.

76 CPA, F. 1 , OP. 8, A.E. 7981 , L. 10-12.

77 CPA, F. 1 , OP. 35 , A.E. 127 et CPA, F. 1, OP. 8, A.E. 8015.

78 OPA, F. 2, OP. 7, A.E. 22, L. 112-113. C'est en 1968 que, d'après certaines sources, Sokrat Markilov de Petrič fonda l'organisation "Ilinden" - archétype du parti macédonien actuel en Bulgarie OMO "Ilinden" : Katardžiev (Ivan), op.cit., p. 367. En 1973, Markilov et un autre activiste important - Stojan Georgiev furent mis en prison - Poulton (Hugh), op.cit., p. 150.

79 CPA, F. 1, OP. 4 O, A.E. 27 O, L. 6. 
Selon les sources documentaires, la grande majorité des participants à ces groupes était constituée par des adolescents qui devaient avoir 20 ans au plus, sans éducation universitaire. Cette " jeunesse turbulente " était, semble-t-il, pour la plupart issue de familles par ailleurs loyales au régime : même des enfants de communistes " ne connaissaient pas " leur "véritable origine " ethnique $^{80}$ ! C'est la raison pour laquelle le Parti répondait à l'activité de ces adolescents en leur accordant - à travers le Komsomol et les directions des lycées une dernière " chance " de se démarquer de leurs " fautes " plutôt qu'en exerçant une violence impitoyable risquant de provoquer une indignation plus générale au sein de la société locale. Mais, mis à part la jeunesse lycéenne, les sources du Parti font également état d'un second groupe social prédisposé à des " actes nationalistes ». Il s'agit d'une partie de l'intelligentsia locale - surtout des instituteurs des écoles des villages - stigmatisés par les fonctionnaires du Parti comme "gens demi-éduqués " (učili-nedoučili) et décrits comme des personnes asociales, des " criminels " ${ }^{81}$. Un membre de la direction centrale du Parti les qualifia de " gens dépravés, ivrognes qui ne font rien [d'utile] dans leur vie "82. Selon le " profil psychologique " élaboré par les fonctionnaires locaux, ce seraient des personnes

avec de grandes prétentions " qui " ont aspiré à beaucoup de choses mais ont atteint peu dans leur vie, pour des raisons subjectives. Ils cherchent, par l'ainsi dite "Question macédonienne ", à réhabiliter leur propre " Je " devant l'opinion publique. On a du mal à travailler avec ce groupe de gens car même s'ils voient la vérité, délibérément, ils ne la reconnaissent pas... ${ }^{83}$

À la différence des adolescents, ces derniers jouissaient de peu de clémence de la part des structures du Parti : en raison de leur nationalisme macédonien, en 1965, plusieurs enseignants des écoles de Kolarovo, Ogražden et Gara Pirin furent licenciés ou envoyés dans d'autres villages ${ }^{84}$. Ils se déclaraient en public " Macédoniens ", tout en cherchant à persuader leurs collègues et voisins à en faire de même. À Samuilovo, la plupart des enseignants n'avaient pas de " positions correctes " (zdravi pozicii) sur la "Question macédonienne " et s'inspiraient des slogans " nationalistes " faits par des lycéens locaux ${ }^{85}$. Dans le cas de ces instituteurs, le choix d'une identification nationale

80 OPA, F. 2, OP. 5, A.E. 23 , L. 85 de 1965 ; OPA, F. 2, OP. 11, A.E. 30 , L. 8-9 de 1978.

${ }^{81}$ OPA, F. 2, OP. 6, A.E. 14 A, L. 19.

82 D'après le haut fonctionnaire Boris Vapcarov - OPA, F. 2. OP. 1, A.E. 164, L. 3.

83 OPA, F. 2, OP. 8 , A.E. 14 , L. 136 de 1969.

84 OPA, F. 2, OP. 5, A.E. 11, L. 70-73 : les instituteurs Pande Paskov, Dimităr Imov (Zajkov), Vangel Tikov, Krăstju Margaritov et d'autres. Voir aussi OPA, F. 2, OP. 4, A.E. 1, L. 4-5, L. 72. La plupart des instituteurs qui déclaraient une identité ethnique macédonienne seraient des districts de Petrič et de Sandanski.

85 OPA, F. 2, OP. 5, A.E. 11, L. 70. 
macédonienne signifiait une marginalisation sociale sûre en Bulgarie communiste. Les leaders actuels de l'organisation OMO " llinden " passèrent des années en prison ou en camps de travail ${ }^{86}$. Certains endroits demeurèrent des " points de tension " à long terme. Bastion de la " propagande de Skopje ", le petit village de Kolarovo, dans le district de Petrič, mérita une attention spéciale de la part du Comité départemental à Blagoevgrad. Le nationalisme macédonien y avait ses adeptes aussi dans les rangs du Parti local lesquels avaient déjà été l'objet des " purges d'agents de Tito " après 1948. Même si toute la direction locale du Parti fut destituée en 1969, Kolarovo restait incurablement " nationaliste ", de même qu'une partie considérable des habitants de Samuilovo et de Skrăt ${ }^{87}$.

Dans les cas des groupes " à risque ", les références romantiques à l'histoire des luttes révolutionnaires en Macédoine de la fin du XIX ${ }^{e}$ et du début du $\mathrm{XX}^{\mathrm{e}}$ siècle et à d'autres époques historiques furent renforcées par l'existence de liens de parenté avec des gens de l'autre côté de la frontière, particulièrement dans les districts frontaliers comme celui de Petric ${ }^{88}$. La population habitant près de la frontière yougoslave bénéficiaient, certains jours de l'année, de la possibilité de visiter la république yougoslave de Macédoine lors des "rencontres frontalières " (pogranični săbori). Le rôle de la diaspora macédonienne constitue également un des facteurs décisifs pour l'apparition d'un nationalisme macédonien dont la nuance pro-yougoslave traduisait aussi une attitude plus générale pro-occidentale ${ }^{89}$. En juin 1970, une enquête sur l'" éducation patriotique et internationale de la population et de la jeunesse " de la région révéla une influence culturelle yougoslave estimée "inquiétante " : $90 \%$ de la population de l'aire de Petrič suivrait régulièrement les émissions de la radio de Skopje et regarderait la télévision du pays voisin ${ }^{90}$. La " mauvaise influence " des médias yougoslaves au sein de la jeunesse du Pirin fut constatée aussi dans les années $1980^{91}$. Selon les autorités du Parti, cette attraction de la Yougoslavie de Tito reposait sur sa prospérité et son " occidentalité " et expliquerait certains cas de "macédonisme ", perçu par les idéologues officiels comme " une invention serbe $n^{92}$. Pour cette raison, au sein

\footnotetext{
86 Par exemple, Jordan Kostadinov, chef de OMO "llinden", fut emprisonné deux fois pour une activité hostile à l'État bulgare : information orale de la part de Kostadinov. Cf. Poulton (Hugh), op.cit., pp. 148-151; Tintoski (Stevan), op.cit., pp. 102-112 ; Katardžiev (Ivan), op.cit., pp. 361-372.

87 OPA, F. 2, OP. 8, A.E. 14, L. 132-165.

88

Cf. OPA, F. 2, OP. 6, A.E. 14A, L. 19-21.

8928 personnes de Kolarovo s'enfuirent, au cours de la collectivisation, en pays occidentaux - OPA, F. 2, OP. 11, A.E. 15, L. 99 , L. 104.
}

91 CPA, F. 1, OP. 4O, A.E. 270, L. 6.

91 OPA, F. 2, OP. 14 , A.E. 32 , L. 8-34.

92 CPA, F. 1, OP. 4O, A.E. 270 , L. 6 ; CPA, F. 1, OP. 40, A.E. 358 , L. 1-2 ; OPA, F. 2, OP. 14 , A.E. 2, L. 65 , de 1970-1971. 
des directions locales du Komsomol à Sandanski et à Gara Pirin, furent fondés certains des premiers " clubs " spéciaux en Bulgarie, clubs destinés au " travail idéologique avec la jeunesse $" 93$.

Parmi les facteurs de "macédonisme ", il faudrait aussi ajouter certains stéréotypes sur les gens du Pirin. Les fonctionnaires du Parti remarquent avec inquiétude que les locaux étaient (et en certains cas sont toujours) stigmatisés dans leur communication avec les autres habitants de la Bulgarie qui employaient à leur égard des étiquettes souvent offensantes, la désignation même de makedonci (Macédoniens) serait porteuse de péjoratifs 94 . Pour renforcer la communication entre la région et le reste du pays, à partir de 1964, parmi les moyens d' " éducation patriotique ", une attention spéciale fut consacrée à l'amélioration de la radiodiffusion et, ensuite, à la construction d'un réémetteur du programme télévisé dans la région suivi par la fondation d'une filiale de la chaîne nationale à Blagoevgrad95.

De même, une série d'investissements furent décidés par la direction du Parti et de l'État pour le " développement économique et culturel " de la région censée représenter un "miroir des succès de la Bulgarie socialiste " 96 . Blagoevgrad, le centre départemental, devenu aussi centre universitaire, fut transformé en une " vitrine " du communisme bulgare destinée à impressionner non seulement les diplomates occidentaux mais aussi les proches de la population du Pirin, habitant de l'autre côté de la frontière97. Le centre de Sandanski subit une reconstruction considérable et la ville fut transformée, en 1981 , en une " station balnéaire d'importance internationale ». Un programme spécial fut destiné à l'aménagement et à la promotion en centre culturel et touristique de la petite ville de Melnik avec le monastère de Rožen, proclamée " ville-musée " (1968) et " ville unique d'importance touristique internationale " (1979). La ville de Bansko, lieu de naissance de personnalités importantes à la fois pour l'histoire bulgare et l'histoire macédonienne, fut proclamée

93 D'après OPA, F. 2, OP. 10, A.E. 9, L. 15-16.

94 OPA, F. 2, OP. 6, A.E. 14A, L. 19-21 ; OPA, F. 2, OP. 14, A.E. 2, L. 226-228. Certains activistes politiques macédoniens en Bulgarie reconnaissent qu'ils " se sentirent " pour la première fois " Macédoniens " lors du service militaire et d'autres cas de première interaction avec des gens de l'intérieur de la Bulgarie - informations orales de militants de OMO "linden" recueillies en avril 2004.

95 Voir CDA, F. 206, OP. 13, A.E. 89 ; CPA, F. 1, OP. 40, A.E. 101, L. 4 ; CPA, F. 1, OP. 4O, A.E. 311, L. 1-2.

96 CPA, F. 1, OP. 6, A.E. 5330 de 1963 ; CPA, F. 1, OP. 35 , A.E. 127 , L. 23 de 1968 ; CPA, F. 1, OP. 35 , A.E. 4773, L. 5-33 de 1974, plus une Résolution du Conseil des ministres de 1975. Cette politique économique devait aussi neutraliser les accusations de la part de Skopje prétendant que la région du Pirin était, par rapport au reste de la Bulgarie, " artificiellement sous-développée ", qu'elle avait un " statut colonial " (cf. Mitrev (Dimităr), op.cit., pp. 290-308.

97 Voir les débats au Comité départemental en février 1983 - OPA, F. 2, OP. 11, A.E. 96, L. 146, L. 149. En 1983 , la filiale de l'Université de Sofia à Blagoevgrad (fondée 1976) fut transformée en Université à part entière. 
centre culturel " d'importance internationale " (1979). Le folklore fut spécialement instrumentalisé dans la promotion du " caractère bulgare " de la région avec le grand festival de chants et de danses populaires Pirin pee (" Le Pirin qui chante ") qui eut lieu en 1962,1967, 1974, 1980, 1985 et continua après. En même temps, les impératifs de l'homogénéisation nationale entraînèrent la " dé-régionalisation " symbolique du Pirin : dans la langue des institutions officielles du Parti et de l'État, la désignation purement administrative " département de Blagoevgrad "(Blagoevgradski okrăg) se substitua au fur et à mesure à la dénomination traditionnelle " Macédoine du Pirin " (Pirinska Makedonija), voire à l'expression plus innocente de " région du Pirin " (Pirinski kraj) ${ }^{98}$.

Blagoevgrad fut visité plusieurs fois par le chef du Parti et de l'État, Todor Živkov. Or, quand il affirma pour la première fois, lors de sa visite en 1962, que la population du Pirin était " patriotique bulgare ", ce slogan provoqua aussi des indignations chez ces derniers 99 . Mais, en juin 1974, il réaffirma, depuis le stade de la ville, le " haut patriotisme " des locaux et annonça devant l'actif du Parti que la "Question macédonienne " n'existait plus dans le département de Blagoevgrad ${ }^{100}$. Et effectivement, dès le début des années 1970, très peu d'" actes nationalistes " suscitèrent l'attention du Comité départemental alors que les complications avec la communauté musulmane orientale du département, administrativement bulgarisée en même temps, restèrent constamment à l'ordre du jour. Les sources documentaires accessibles aux archives du Parti et d'État ne font pas montre d'opposition importante à la politique d' " éducation nationale " dans le reste de la population et viennent attester le caractère plutôt marginal des cas de résistances et de revendications ethniques macédoniennes. Déjà en 1969, les autorités locales du Parti se félicitent de la " victoire " de leur politique nationale anti-macédoniste dans toute la région sauf dans quelques villages isolés comme Kolarovo ${ }^{101}$. Huit ans plus tard, l'" éducation patriotique " suscitait de nouveau un Plénum spécial du Comité départemental qui eut lieu en novembre 1977 mais, cette fois-ci, les manifestations de nationalisme macédonien étaient à peine remarquées ${ }^{102}$. Aussi, en juin 1978, le public de Blagoevgrad acclama-t-il sans ressentiment particulier

98 Processus remarqué par Troebst (Stefan), op.cit. [trad. macédonienne Trebst (Stefan), BugarskoJugoslovenskata kontroverza za Makedonija 1967-1982, Skopje, INI, 1997, pp. 158-159].

99 Cf. Germanov (Stojan), art.cit., p. 125 .

100 OPA, F. 2, OP. 10, A.E. 18, L. 66. Petrič fut proclamé par Živkov " forteresse de la bulgarité " (krepost na bălgarštinata) - Ibid., p. 79-80.

${ }^{101}$ OPA, F. 2, OP. 8, A.E. 14, L. 165.

102 OPA, F. 2, OP. 11 , A.E. 15. 
un nouveau discours de Živkov où le Pirin fut proclamé " symbole de l'intégrité et d'indivisibilité de la mère Bulgarie (majka Bălgarija), chair de la chair

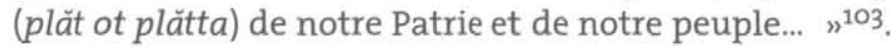

\section{CONCLUSION}

La " renaissance " d'un mouvement identitaire macédonien dans la région à partir de la chute du régime communiste en 1989 et au début des années 1990 correspond à une poussée à l'échelle mondiale du militantisme national macédonien représenté par des dizaines d'ONG en République de Macédoine, en Grèce, en Albanie, mais aussi en Australie, au Canada etc. ${ }^{104}$. Les dernières années marquent cependant une nouvelle baisse des revendications minoritaires macédoniennes en Bulgarie. Ce fait ne serait pas compréhensible dans le cadre figé de la notion essentialiste de l'identité ethnique employée par un bon nombre de scientifiques et de militants d'ONG transnationales instrumentalisant le discours multiculturaliste sur les droits des minorités. Bien entendu, on ne peut nier la répression du militantisme politique macédonien pendant ou après le communisme à laquelle les leaders actuels du parti OMO " Ilinden " font référence. Pourtant, les concepts d'" assimilation " ou d'" acculturation " ne sauraient contribuer à la compréhension des phénomènes identitaires dans le Pirin.

Selon le paradigme de "frontière ethnique " proposé par Fredrick Barth ${ }^{105}$, l'ethnicité n'est qu'un mode d'(auto-)identification et, loin de renvoyer à une " essence " du groupe, il est actualisé au sein de l'interaction sociale dans des circonstances précises. C'est le contexte de cette interaction qui détermine la pertinence ou la saillance d'une identité ou d'une autre dans les diverses situations ainsi mises en jeu. L'identité ethnique, dans cette perspective, ne doit aucunement être perçue comme une entité immuable : elle est, au contraire, définie dans le champ de forces d'ordre politique, social ou culturel, sources d'impact ou de violence symbolique sur les modes d'auto-identification $^{106}$. Le cas de la Macédoine du Pirin ne fait que procurer davantage d'argu-

\footnotetext{
${ }^{103}$ Voir Živkov (Todor), Izbrani săčinenija, Sofia : Tom 27, Sofia, 1980, p. 415.

104 Processus analysé par Danforth (Loring), The Macedonian Conflict. Ethnic Nationalism in a Transnational World, Princeton : Princeton University Press, 1995.

105 Cf. Barth (Fredrick), ed., Ethnic Groups and Boundaries : The Social Organization of Cultural Difference, Bergen / Oslo : Universitetsforlaget, 1969.

${ }^{106}$ Peter Sahlins, par exemple, élabora une analyse de la façon dont le maintien des frontières d'exclusion et/ou d'inclusion déterminait les formes et les expressions des différentes identités collectives dans le cadre restreint d'une petite communauté frontalière partagée entre la France et l'Espagne. Cf. Sahlins (Peter), Boundaries. The Making of France and Spain in the Pyrénées, Berkeley : University of California Press, 1989 .
} 
ments pour une perspective conceptuelle de ce type. La création d'une république macédonienne en Yougoslavie titiste qui fournit le cadre politique indispensable pour l'affirmation complète du nationalisme ethnique macédonien d'un côté, et d'un autre, la politique homogénéisatrice intransigeante de la Bulgarie de Živkov, firent apparaître une frontière ethnique au sein de la région du Pirin qui n'existait pas auparavant. Mais cette frontière n'est pas fixe, elle continue à osciller et à constituer l'identité des " Bulgares du département de Blagoevgrad " et simultanément celle des " Macédoniens du Pirin "107. 